\title{
On the low-energy behavior of the Adler function
}

\author{
A.V. Nesterenko ${ }^{\text {a* }}$ \\ ${ }^{a}$ BLTPh, Joint Institute for Nuclear Research, Joliot Curie 6, Dubna, 141980, Russian Federation
}

The infrared behavior of the Adler function is examined by making use of a recently derived integral representation for the latter. The obtained result for the Adler function agrees with its experimental prediction in the entire energy range. The inclusive $\tau$ lepton decay is studied in the framework of the developed approach.

\section{INTRODUCTION}

The Adler function 1 plays a key role in particle physics. Specifically, theoretical description of some strong interaction processes (e.g., electronpositron annihilation into hadrons [2] and inclusive $\tau$ lepton decay 34) is inherently based on this function. Besides, Adler function is essential for confronting the precise experimental measurements of some electroweak observables (e.g., muon anomalous magnetic moment [5] and shift of the electromagnetic fine structure constant [6]) with their theoretical predictions. In turn, the latter represents a decisive test of the Standard Model and imposes strict restrictions on possible "new physics" beyond it.

Furthermore, Adler function plays a crucial role for the congruous analysis of spacelike and timelike experimental data. Indeed, since perturbation theory and renormalization group method are not applicable directly to the study of observables depending on the timelike kinematic variable, for the self-consistent description of the latter one has to relate the timelike experimental data with the spacelike perturbative results. Here, the required link between the experimentally measurable $R$-ratio of electron-positron annihilation into hadrons and theoretically computable Adler function $D\left(Q^{2}\right)$ is represented by the dispersion relation [1]

$D\left(Q^{2}\right)=Q^{2} \int_{4 m_{\pi}^{2}}^{\infty} \frac{R(s)}{\left(s+Q^{2}\right)^{2}} d s$

where $m_{\pi} \simeq 135 \mathrm{MeV}$ [7] stands for the mass of the lightest hadron state. The dispersion rela-

*E-mail: nesterav@theor.jinr.ru tion (1) is also commonly employed for extracting the Adler function from the relevant experimental data. For this purpose, in the integrand (1) $R(s)$ is usually parameterized by its experimental measurements at low and intermediate energies and by its theoretical prediction at high energies.

The ultraviolet behavior of the Adler function can be approximated by the power series in the strong running coupling within the perturbation theory (see paper [8] and references therein)

$D_{\text {pert }}^{(\ell)}\left(Q^{2}\right)=1+\sum_{j=1}^{\ell} d_{j}\left[\alpha_{\mathrm{s}}^{(\ell)}\left(Q^{2}\right)\right]^{j}$.

The overall factor $N_{\mathrm{c}} \sum_{f} Q_{f}^{2}$ is omitted throughout, where $N_{\mathrm{c}}=3$ is the number of colors and $Q_{f}$ denotes the charge of the quark of the $f$-th flavor. In Eq. (2) $\alpha_{\mathrm{s}}^{(\ell)}\left(Q^{2}\right)$ is the $\ell$-loop perturbative QCD invariant charge, $\alpha_{\mathrm{s}}^{(1)}\left(Q^{2}\right)=4 \pi /\left(\beta_{0} \ln z\right)$, $z=Q^{2} / \Lambda^{2}, \beta_{0}=11-2 n_{\mathrm{f}} / 3, n_{\mathrm{f}}$ is the number of active quarks, and $d_{1}=1 / \pi$.

However, the perturbative expansion (2) is invalid at low energies and it is inconsistent with the dispersion relation for the Adler function (1) due to unphysical singularities of the strong running coupling $\alpha_{\mathrm{s}}\left(Q^{2}\right)$ in the infrared domain. The latter also causes certain difficulties in processing the low-energy experimental data.

\section{NOVEL INTEGRAL REPRESENTA- TION FOR THE ADLER FUNCTION}

In general, there is a variety of the nonperturbative approaches to handle the strong interaction processes at low energies. In this work we will focus on the approach which engages dispersion relations. Indeed, the latter provide an im- 
portant source of the nonperturbative information about the hadron dynamics in the infrared domain, which should certainly be taken into account when one is trying to go beyond the scope of perturbation theory.

In particular, dispersion relation (10) imposes stringent physical nonperturbative constraints on the Adler function. Specifically, since $R(s)$, being the ratio of two cross-sections, assumes finite values and tends to a constant in the ultraviolet asymptotic $s \rightarrow \infty$, then the Adler function $D\left(Q^{2}\right)$ vanishes 1 in the infrared limit $Q^{2}=0$. In addition, dispersion relation (1) implies that the Adler function possesses the only cut $Q^{2} \leq$ $-4 m_{\pi}^{2}$ along the negative semi-axis of real $Q^{2}$.

These nonperturbative constraints on the Adler function have been merged with its perturbative approximation in Refs. 910] (see also discussion of this issue in Ref. [11]). Eventually, this results in the following integral representations for the Adler function and $R$-ratio:

$$
\begin{aligned}
D\left(Q^{2}\right) & =\frac{Q^{2}}{Q^{2}+4 m_{\pi}^{2}}\left[1+\int_{4 m_{\pi}^{2}}^{\infty} \rho(\sigma) \frac{\sigma-4 m_{\pi}^{2}}{\sigma+Q^{2}} \frac{d \sigma}{\sigma}\right], \\
R(s) & =\theta\left(s-4 m_{\pi}^{2}\right)\left[1+\int_{s}^{\infty} \rho(\sigma) \frac{d \sigma}{\sigma}\right],
\end{aligned}
$$

where $\theta(x)$ is the unit step function, $\theta(x)=1$ if $x \geq 0$ and $\theta(x)=0$ otherwise. The developed approach [9] eliminates such intrinsic difficulties of perturbation theory as the infrared unphysical singularities of outcoming results. Besides, additional parameters are not introduced into the theory. Furthermore, Eq. (4) by construction accounts for the effects due to the analytic continuation of spacelike theoretical results into timelike domain, such as the resummation of the so-called $\pi^{2}$-terms. It is worth noting also that the mass of the lightest hadron state affects both the parton model prediction and the strong correction of the quantities in hand (3), (4).

In the limit of the massless pion $m_{\pi}=0$ expressions (3) and (4) become identical to those of the Analytic Perturbation Theory (APT), see papers 1213] and references therein. However, it is

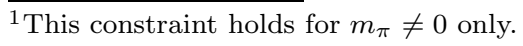

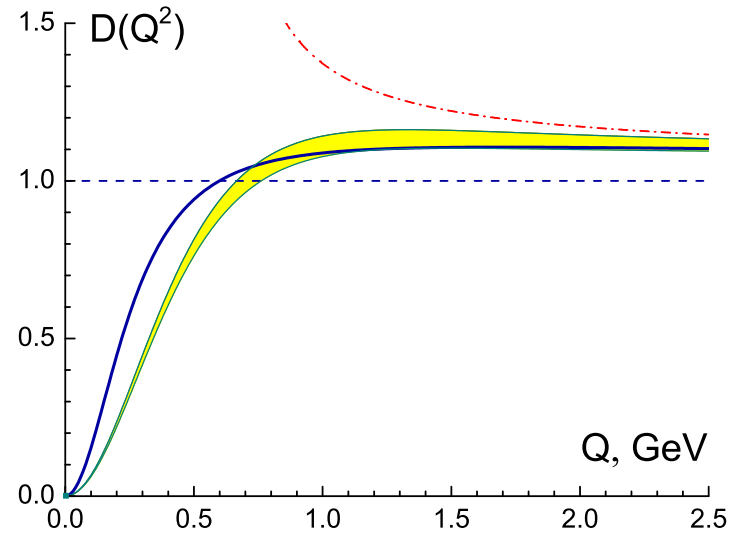

Figure 1. Adler function (3) corresponding to the spectral density (6) (solid curve) $(\Lambda=441 \mathrm{MeV}$, $n_{\mathrm{f}}=3$ ). Perturbative approximation (2) of the Adler function and its experimental prediction are denoted by the dot-dashed curve and shaded band, respectively.

crucial to keep the pion mass nonvanishing, since it can be safely neglected only when one handles the strong interaction processes at high energies. It is worth mentioning that there is a number of other similar approaches 2 which also combine perturbative results with relevant dispersion relations, see, e.g., Refs. 15/16/17/18/19.

The spectral density $\rho(\sigma)$, which appears in Eqs. (3) and (4), can be determined either as the discontinuity of the explicit "exact" theoretical expression for the Adler function $D_{\text {exact }}\left(Q^{2}\right)$ across the physical cut or as the numerical derivative of the experimental data on $\mathrm{R}$-ratio 9 :

$\rho(\sigma)=\frac{1}{\pi} D_{\text {exact }}\left(-\sigma+i 0_{+}\right)=-\frac{d R_{\exp }(\sigma)}{d \ln \sigma}$.

However, there is still no explicit "exact" expression for the Adler function, and, therefore, there is no unique way to compute the corresponding spectral density (5) by making use of its approximate perturbative expression (21). In what follows we will employ the spectral function obtained in

\footnotetext{
${ }^{2}$ The Adler function was studied in the framework of APT supplemented with the relativistic quark mass threshold resummation in Ref. 14 .
} 
Ref. $203^{3}$, which has the following form at the one-loop level [20]22]:

$\rho^{(1)}(\sigma)=\left(1+\frac{\Lambda^{2}}{\sigma}\right) \frac{1}{\ln ^{2}\left(\sigma / \Lambda^{2}\right)+\pi^{2}}$.

The Adler function (3), corresponding to the spectral function (6), is presented in Fig. (1) by solid curve. The dot-dashed curve stands for the one-loop perturbative approximation (2) of the Adler function, whereas its experimental prediction, computed in the way described above, is denoted by the shaded band. As one may infer from Fig. 11 the obtained result for the Adler function is in a reasonable agreement with its experimental prediction in the entire energy range.

\section{INCLUSIVE $\tau$ LEPTON DECAY}

It is also of a particular interest to study the inclusive $\tau$ lepton decay within the approach in hand, since this process probes the infrared hadron dynamics at energies below the mass of the $\tau$ lepton, and the relevant experimental data are fairly precise. The measurable quantity here is the inclusive semileptonic branching ratio

$$
R_{\tau}=\frac{\Gamma\left(\tau^{-} \rightarrow \text { hadrons }^{-} \nu_{\tau}\right)}{\Gamma\left(\tau^{-} \rightarrow e^{-} \bar{\nu}_{e} \nu_{\tau}\right)}
$$

which can be split into three parts, namely, $R_{\tau}=$ $R_{\tau, \mathrm{V}}+R_{\tau, \mathrm{A}}+R_{\tau, \mathrm{S}}$. The terms $R_{\tau, \mathrm{v}}$ and $R_{\tau, \mathrm{A}}$ account for the contributions to Eq. (7) of the decay modes with the light quarks only, and they correspond to the vector $(\mathrm{V})$ and axial-vector $(\mathrm{A})$ quark currents, respectively. The last term $R_{\tau, \mathrm{s}}$ accounts for the contribution to Eq. (7) of the decay modes with the $s$ quark.

Let us proceed with the nonstrange part of the ratio (7) associated with the vector quark currents

$R_{\tau, \mathrm{v}}=\frac{N_{\mathrm{c}}}{2}\left|V_{\mathrm{ud}}\right|^{2} S_{\mathrm{EW}}\left(\Delta_{\mathrm{QCD}}+\delta_{\mathrm{EW}}^{\prime}\right)$,

see papers [3] and references therein for detailed discussion of this issue. The experimental measurement [23] of the ratio (8) yields $R_{\tau, \mathrm{v}}=$

\footnotetext{
${ }^{3}$ It is interesting to note that the QCD effective coupling obtained in Ref. 20] has been independently rediscovered in Ref. 21] proceeding from entirely different reasoning.
}

1.764 \pm 0.016 . In Eq. (8) $\left|V_{\text {ud }}\right|=0.97418 \pm 0.00027$ denotes the Cabibbo-Kobayashi-Maskawa matrix element [7, $S_{\mathrm{EW}}=1.0194 \pm 0.0050$ and $\delta_{\mathrm{EW}}^{\prime}=$ 0.0010 are the electroweak corrections [3]24, and $\Delta_{\mathrm{QCD}}$ can be expressed in terms of a weighted integral of the aforementioned $R(s)$-ratio:

$\Delta_{\mathrm{QCD}}=2 \int_{0}^{M_{\tau}^{2}}\left(1-\frac{s}{M_{\tau}^{2}}\right)^{2}\left(1+2 \frac{s}{M_{\tau}^{2}}\right) R(s) \frac{d s}{M_{\tau}^{2}}$,

where $M_{\tau} \simeq 1.777 \mathrm{GeV}[7]$ is the $\tau$ lepton mass.

In the framework of perturbative approach one usually reduces Eq. (9) to the contour integral in the complex $s$-plane along the circle of the radius of the squared mass of the $\tau$ lepton. At the oneloop level this eventually leads to 3 .

$\Delta_{\mathrm{QCD}}=1+d_{1} \alpha_{\mathrm{s}}^{(1)}\left(M_{\tau}^{2}\right)$,

that, in turn, results in $\Lambda=(678 \pm 55) \mathrm{MeV}$ for $n_{\mathrm{f}}=2$ active quarks.

At the same time, for the evaluation of $\Delta_{\mathrm{QCD}}$ in the framework of the approach in hand, the integration in Eq. (9) can be performed in a straightforward way. Ultimately this leads to the following result at the one-loop level [10]:

$$
\begin{aligned}
\Delta_{\mathrm{QCD}} & =1-\delta_{\Gamma}+d_{1} \alpha_{\mathrm{TL}}^{(1)}\left(M_{\tau}^{2}\right)-d_{1} \delta_{\Gamma} \alpha_{\mathrm{TL}}^{(1)}\left(m_{\Gamma}^{2}\right) \\
& +d_{1} \frac{4 \pi}{\beta_{0}} \int_{\chi}^{1} f(\xi) \rho^{(1)}\left(\xi M_{\tau}^{2}\right) d \xi,
\end{aligned}
$$

where $f(\xi)=\xi^{3}-2 \xi^{2}+2, \chi=m_{\Gamma}^{2} / M_{\tau}^{2}, \delta_{\Gamma}=$ $\chi f(\chi)$, and

$\alpha_{\mathrm{TL}}^{(1)}(s)=\frac{4 \pi}{\beta_{0}} \theta\left(s-m_{\Gamma}^{2}\right) \int_{s}^{\infty} \rho^{(1)}(\sigma) \frac{d \sigma}{\sigma}$

is the one-loop timelike effective coupling [9]. Here $m_{\Gamma}$ stands for the total mass of the lightest allowed hadronic decay mode of the $\tau$ lepton, e.g., for the vector channel $m_{\Gamma}=m_{\pi^{0}}+m_{\pi^{-}}$. In this case $\delta_{\Gamma} \simeq 0.048$ considerably exceeds the electroweak correction $\delta_{\mathrm{EW}}^{\prime}$. Eventually, Eq. (11) results in $\Lambda=(941 \pm 86) \mathrm{MeV}$ for $n_{\mathrm{f}}=2$ active quarks, that is somewhat larger than the one-loop perturbative estimation quoted above.

The effects due to the nonvanishing hadronic mass $m_{\Gamma}$ play a substantial role herein. In particular, in the massless limit $m_{\Gamma}=0$ Eq. (11) leads to $\Lambda=(493 \pm 56) \mathrm{MeV}$ for $n_{\mathrm{f}}=2$ active quarks. 


\section{SUMMARY}

The infrared behavior of the Adler function is studied by making use of recently derived integral representation for the latter. The developed approach possesses a number of appealing features. Namely, it eliminates unphysical perturbative singularities, properly accounts for the effects due to the analytic continuation of spacelike theoretical results into timelike domain, and embodies the effects due to the mass of the lightest hadron state. Besides, additional adjustable parameters are not introduced into the theory. Furthermore, the developed approach provides a reasonable description of the Adler function in the entire energy range. It is also shown that the effects due to the nonvanishing mass of the lightest hadron state play a substantial role in processing the experimental data on the inclusive $\tau$ lepton decay.

\section{Acknowledgements}

This work was partially performed during the visit of the author to the University of Milano. Author is thankful to Professor Giovanni Prosperi for his kind hospitality and fruitful discussions. Author is grateful to N. Brambilla, S. Forte, and A. Vairo for the interest to this study. Partial financial support of grants RFBR-08-01-00686, BRFBR-JINR-F08D-001, and NS-1027.2008.2 is acknowledged.

\section{REFERENCES}

1. S.L. Adler, Phys. Rev. D 10, 3714 (1974).

2. R.P. Feynman, Photon-Hadron Interactions (Benjamin, Massachusetts, 1972).

3. E. Braaten, S. Narison, and A. Pich, Nucl. Phys. B 373, 581 (1992).

4. M. Davier, S. Descotes-Genon, A. Hocker, B. Malaescu, and Z. Zhang, arXiv:0803.0979 [hep-ph].

5. M. Davier and W.J. Marciano, Ann. Rev. Nucl. Part. Sci. 54, 115 (2004); M. Passera, J. Phys. G 31, R75 (2005); J.P. Miller, E. de Rafael, and B.L. Roberts, Rept. Prog. Phys. 70, 795 (2007).

6. F. Jegerlehner, arXiv:0807.4206 [hep-ph].

7. C. Amsler et al. [Particle Data Group], Phys. Lett. B 667, 1 (2008).
8. P.A. Baikov, K.G. Chetyrkin, and J.H. Kuhn, Phys. Rev. Lett. 101, 012002 (2008).

9. A.V. Nesterenko and J. Papavassiliou, J. Phys. G 32, 1025 (2006); A.V. Nesterenko, arXiv:0710.5878 [hep-ph].

10. A.V. Nesterenko, in preparation.

11. M. Baldicchi, A.V. Nesterenko, G.M. Prosperi, D.V. Shirkov, and C. Simolo, Phys. Rev. Lett. 99, 242001 (2007); M. Baldicchi, A.V. Nesterenko, G.M. Prosperi, and C. Simolo, Phys. Rev. D 77, 034013 (2008).

12. D.V. Shirkov and I.L. Solovtsov, Phys. Rev. Lett. 79, 1209 (1997); Phys. Lett. B 442, 344 (1998); Theor. Math. Phys. 150, 132 (2007).

13. D.V. Shirkov, Eur. Phys. J. C 22, 331 (2001); Nucl. Phys. B (Proc. Suppl.) 152, 51 (2006); arXiv:0807.1404 [hep-ph].

14. K.A. Milton, I.L. Solovtsov, and O.P. Solovtsova, Phys. Rev. D 65, 076009 (2002); 64, 016005 (2001); Mod. Phys. Lett. A 21, 1355 (2006).

15. A.P. Bakulev, S.V. Mikhailov, and N.G. Stefanis, Phys. Rev. D 75, 056005 (2007); 77, 079901(E) (2008); Phys. Rev. D 72, 074014 (2005); 72, 119908(E) (2005).

16. A.I. Alekseev and B.A. Arbuzov, Mod. Phys. Lett. A 20, 103 (2005); 13, 1747 (1998).

17. D.M. Howe and C.J. Maxwell, Phys. Rev. D 70, 014002 (2004); Phys. Lett. B 541, 129 (2002).

18. I. Caprini and J. Fischer, Phys. Rev. D 71, 094017 (2005); 60, 054014 (1999); Eur. Phys. J. C 24, 127 (2002).

19. G. Cvetic and C. Valenzuela, J. Phys. G 32, L27 (2006); arXiv:0804.0872 [hep-ph].

20. A.V. Nesterenko, Phys. Rev. D 62, 094028 (2000); 64, 116009 (2001).

21. F. Schrempp, J. Phys. G 28, 915 (2002); D. Klammer and F. Schrempp, JHEP 0806, 098 (2008).

22. A.V. Nesterenko, Nucl. Phys. B (Proc. Suppl.) 133, 59 (2004); Int. J. Mod. Phys. A 18, 5475 (2003).

23. K. Ackerstaff et al. (OPAL Collaboration), Eur. Phys. J. C 7, 571 (1999).

24. W.J. Marciano and A. Sirlin, Phys. Rev. Lett. 61, 1815 (1988); E. Braaten and C.S. Li, Phys. Rev. D 42, 3888 (1990). 